\title{
Selecting SaaS CRM Solution for SMEs
}

\author{
Oyepeju Oyekola ${ }^{\dagger}$ \\ Faculty of Science and Technology \\ Bournemouth University \\ United Kingdom \\ i7433055@bournemouth.ac.uk
}

\author{
Lai $\mathrm{Xu}^{\dagger}$ \\ Faculty of Science and Technology \\ Bournemouth University \\ United Kingdom \\ lxu@bournemouth.ac.uk
}

\begin{abstract}
The use of CRM system has helped many organisations to manage and analyze business interaction with customers in several ways. However, Small and Medium-sized Enterprises (SMEs) are often struggling to find an optimal solution to fit their business and financial conditions. When faced with different SaaS-based CRM solutions, SMEs need to save investments in IT infrastructure, the cost of CRM deployment, and the maintenance cost. To select the best solution for the SME is critical for the business. This paper look at What systematic approach SMEs needs to follow when choosing the best SaaS CRM solution that would suit their company business strategy? To answer this question, this study presents a comprehensive decision-making framework for SaaS CRM evaluation and selection approach using the Analytical Hierarchical Process (AHP). The proposed selection approach is also applied to an SME, Biyemfat Enterprise, a small private real estate company. A case study of selecting a SaaS CRM solution for Biyemfat Enterprise is presented with an evaluation of the cost-benefit analysis.
\end{abstract}

\section{KEYWORDS}

CRM systems, SaaS CRM solutions, Multiple Criteria Decision Making (MCDM), Analytical Hierarchical Process (AHP), Costbenefit analysis.

\section{Introduction}

Recent trends such as increasing competition, rising customer expectation, technological innovation and markets expansion have forced enterprises to change their orientation of delivery activities and value creation from product-centric culture to customercentric model [1]. As a result, organizations have resorted to stateof-the-art information technologies in managing the customer relationship and formulating well-defined customer-centric strategies that support such initiatives [2]. More than ever, delivering a positive customer experience at every opportunity requires a much greater level of planning and cross-organizational coherence. In view of this, the concept of Customer Relationship Management (CRM) system has become a vital tool for improving customer lifetime value or delivering differentiated and superior customer experience [3].

With the advancement in technology, software providers are able to offer a variety of customizable CRM solution packages to meet customer demand and also offering them a chance to choose from different deployment options such as the traditional/on-premise
CRM deployment or SaaS CRM deployment [4] [5]. The shift to cloud computing service model (SaaS) have disrupted the CRM market to a large extent, creating new opportunities for vendors and supporting organisations with their business processes, offering them a range of options when deciding on deployment such as the single or multi-tenant subscription-based models as part of their portfolio of CRM solutions [6]. The subscriptionbased licensing and hosted multi-tenant models allow organizations to pay for using the software on a subscription basis, access and store customer information via the internet and hosted on a third-party server.

Compare to the traditional CRM deployment usually implemented by large organisations with strong financial capability; CRM software vendors now prioritize the deployment of SaaS CRM in order to encourage businesses of all sizes [7]. And most recently, the adoption of SaaS CRM solution is rapidly increasing among SMEs to provide better customer service and improve efficiency [8]. Factors such as low operating cost, increased flexibility, faster time to market, competitive advantage, less support on technical expertise and expansion of market opportunities are just a few of the drivers behind the acceptance of SaaS a viable CRM deployment option [9].

With a range of SaaS CRM solutions to choose from with different purposes, configuration and prices; SMEs are put off by the number of times needed to evaluate each one and the fear of choosing the wrong solution. As the selection of the wrong SaaS CRM solution could lead to a wrong strategic decision and great financial loss to the organisation [10]. Also, running SaaS-based CRM solutions is different from the traditional CRM package solution, the selection criteria are thus different. This uncertainty is further mystified by the fact that no existing SaaS-based CRM solution can meet all business requirement or company functionalities [11] [12]. Therefore, it is extremely important for organisations to examine its core business strategy and ensure that the business strategy is supported by feasible and appropriate technology architecture [13]. A vital element for attaining this is to have a careful analysis of organizational processes. Such an approach would essentially guide towards developing an appropriate roadmap that is responsive to customer needs before making the decision of selecting a SaaS CRM solution.

This paper presents a step by step approach used as a complete guide to supporting SMEs in the evaluation and selection of SaaS CRM solutions. The proposed method ensures that the evaluation process is aligned with business strategies and goals. To 
demonstrate the practical viability of the model a small real estate business will be used as a case study. The structure of the paper is as follows: Section 2 discusses selection criteria that can be used to evaluate SaaS CRM solution based on literature. Section 3 describes the selection method that can be adopted by SMEs. Section 4 describe the case study. Section 5 describes the implementation of the proposed method with the case study in Section 4. Section draws the conclusion of this paper, its limitation, significance and future study.

\section{Selection Criteria}

Selection criteria are a crucial key for implementing CRM for an organisation. It requires a full examination of many contradictory factors and very difficult to evaluate when selecting CRM solutions. The most complex process of selection is the strategic aspect. Evidence has suggested that realizing greater value from CRM implementation is highly reliant upon the capability of the solutions to achieve the company's overall CRM strategy, deciding to select a solution over another should be based on a wide range of factors such as involving the right people, the degree of alignment between the solution's features and the specific objectives of the company's CRM strategy among others [14]

Few studies have attempted to address the issue of identifying the criteria's for evaluating and selecting the most suitable CRM solution. For instance, Colombo and Francalanci [15] describe a hierarchical ranking model for CRM selection packages based on their functional and technical quality. Oztaysi, Kaya, and Kahraman [16] compare the CRM performance of e-commerce firms using the ANP approach. Keramati and Sangari [17] proposed a success framework to identify associated critical factors of CRM using a Fuzzy ANPP Approach. Lee, Tang and Sugumaran [18]uses AHP process to develop a decision-making framework for selecting the best open source CRM software. However, their study is limited and do not explain how the company strategic objectives can be used to extract proper selection criteria into a fundamental hierarchy for evaluating SaaS CRM solution. Consequently, this study reviewed several literatures and identify some key criteria that must be considered when selecting most suitable SaaS CRM solution in Table 1. And more importantly, this study combined the criteria for both SaaS product selection and CRM selection criteria.

\begin{tabular}{|c|c|c|}
\hline Criteria & Related Literature & Description \\
\hline Total cost & $\begin{array}{l}{[19][20],[21],[22]} \\
{[18],[23][14]}\end{array}$ & $\begin{array}{l}\text { This denotes the total cost that a company spends on the implementation of a } \\
\text { SaaS CRM solution }\end{array}$ \\
\hline Functionality & $\begin{array}{l}{[21][24],[25][26],} \\
{[25][14]}\end{array}$ & $\begin{array}{l}\text { This denote the degree at which the solution satisfies stated company needs in } \\
\text { terms of SaaS CRM such as Salesforce automation (Account contact } \\
\text { management, Track interaction/reporting, Schedules and reminders, contract } \\
\text { management), Market automation (Email marketing, web lead management), } \\
\text { Analytical CRM, Service automation }\end{array}$ \\
\hline Usability & $\begin{array}{l}{[21][24][18][23],} \\
{[27]}\end{array}$ & $\begin{array}{l}\text { Usability refers to the degree at which an organisations uses software solution } \\
\text { with effectiveness, efficiency and ease. This can be broken down into friendly } \\
\text { GUI, and Mobility }\end{array}$ \\
\hline Reliability & $\begin{array}{l}{[28],[19],[21][22]} \\
{[18],[23],[26][27]}\end{array}$ & $\begin{array}{l}\text { It is important to know the extent to which the software is reliable and protected } \\
\text { It also an evidence to show business continuity from the vendor. It is sub- } \\
\text { divided into security/recovery and stability }\end{array}$ \\
\hline Fit with company & {$[18]$} & $\begin{array}{l}\text { Companies need to understand in the earlier stage if the software solution is fit } \\
\text { for their business strategy, if it is appropriate for their company management. It } \\
\text { has three sub characteristics: Business goals, industry and capabilities. }\end{array}$ \\
\hline Flexibility & {$[22][18]$} & $\begin{array}{l}\text { This explains how flexible SaaS CRM solution can be. For instance, an ability } \\
\text { to easily support changes in company strategy and policy and to check whether } \\
\text { it can adapt to the constant change in business world. It is broken down into } \\
\text { customization, compatibility, scalability and ease of integration. }\end{array}$ \\
\hline Vendor quality & {$[21][18]$} & $\begin{array}{l}\text { The reputation or the popularity of SaaS CRM system provider partly reveals } \\
\text { vendor product capability and ability. For instance, the number of downloads } \\
\text { the vendor has, support provided from present customers, their product market } \\
\text { share, awards won could be used to evaluate the vendor reputation. This is } \\
\text { divided into vendor reputation and technical support }\end{array}$ \\
\hline Service quality & [24] & $\begin{array}{l}\text { This describe the SLA (Service Level Agreement), continuity plan, data } \\
\text { management and migration plan. }\end{array}$ \\
\hline Product triability & [19], & $\begin{array}{l}\text { The ability for the vendors to provide a free trials and demos for customers } \\
\text { before sale }\end{array}$ \\
\hline $\begin{array}{l}\text { Vendor } \\
\text { observability }\end{array}$ & [19], & $\begin{array}{l}\text { This indicates whether the vendor can create trust with the customer by } \\
\text { showcasing the success stories with successful case studies and prove their } \\
\text { domain knowledge before the purchase. }\end{array}$ \\
\hline Integration & [21]] & $\begin{array}{l}\text { The ability of the system to easily integrate with existing legacy systems of the } \\
\text { customer }\end{array}$ \\
\hline
\end{tabular}

Table 1: Selection Criteria

\section{Method for Selecting SaaS CRM solution for SMEs}

This section presents a step by step approach used as a guide to support SMEs in the selection of SaaS CRM solution making sure the evaluation process is aligned with the business strategies and goals. The method consists of three stages which are Preliminary, Evaluation and Configuration stage as shown in Figure 1. Detailed descriptions of the preliminary stage are presented in Section 3.1. The evaluation stage uses the AHP evaluation method to quantify different selectors in Section 3.2. Finally, the configuration stage is also explained in Section 3.3. The method involves an iterative process in which the result of one stage will determine whether to move on to the next stage. Possible changes and refinement can be considered at each stage of the proposed method or a decision to opt-out. 


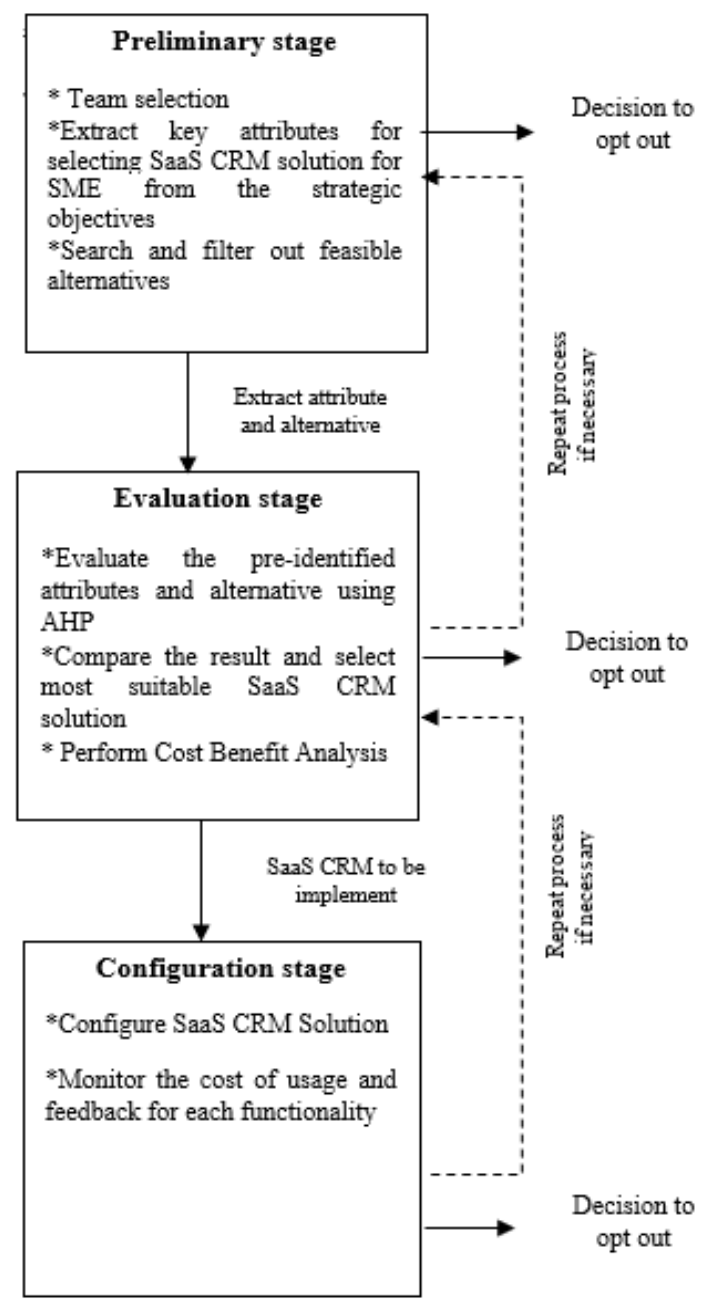

Figure 1: Comprehensive SaaS-based CRM Selection Framework

\subsection{Preliminary Stage}

The purpose of this stage to identify what goals the company want to achieve through SaaS CRM. Identifying the company-specific needs and then researching on possible feasible SaaS CRM solutions will help to determine which of the SaaS CRM solutions provide the most complete solutions. Three different tasks are involved in the preliminary stage, namely team selection, extracting key attributes from the strategic objective, and filter out a feasible alternative. The first step involves team selection. Selection of team helps to criticize any bias or single-person point of view and basically takes in the view point and experience of the whole team in the selection and evaluation process. As smaller organizations use a more centralized form of decision-making with fewer people involved in the selection process [32]. Then, select a team consisting of a CRM expert, Staff person and at least one decision-maker from the company. During this time, the team describe the project characteristics such as problem definition, company goals, and any risk or concerns.

Secondly, the team brainstorm and identify the strategic objective and ensure its scope is appropriate for the project characteristics.
It is worth noting that the project characteristics need to clearly define the company needs as improper direction and strategy might not bring optimal results. Identifying the strategic objectives guide the main goal that the team strive to achieve and can be used to extract the proper criteria before incorporating them into a fundamental objectives' hierarchy. In order to incorporate the objectives into a hierarchical structure, a top-down decomposition will be used. The overarching goals are to select a suitable SaaS CRM solution. The team will drill down the strategic objectives to obtain the proper criteria for evaluation and this represent the top-level of the fundamental objectives. For instance, asking the team "what the identified objective means" and "How do we achieve them". The top-level will further be decomposed to extract the attributes for evaluating the SaaS CRM solution which is represented at the lower-level fundamental objective. The identified attribute will be consistent with the objective framework which is guided by the company strategy [33].

Thirdly, CRM expert research SaaS CRM market based on the company business requirement. This is done by obtaining a wide range of information relating to SaaS CRM solution vendors through the internet, product/service release conferences, professional magazines, vendor websites, and other sources. This helps to have a strong background of each of the alternatives and make good comparisons. The potential alternatives will be filtered out to about 2-4 feasible alternatives through a thorough examination and evaluation of live demos or free trials.

\subsection{Evaluation Stage}

The second stage is the Evaluation stage and it involves using AHP evaluation method. The AHP is a decision-making model used to solve complex multi-criteria decision-making problems involving priority setting, planning, alternative selection and resource allocation. It involves three steps: decomposition step, comparative judgement step and synthesis of priority step [33] [34]. The decomposition step involves constructing a structural hierarchy of the decision problem from the fundamental hierarchy as discussed in the preliminary stage. The comparative judgement step involves setting up a matrix to obtain the pairwise comparisons for the attributes and alternatives using a nine-point scale and then determine the consistency of the inputted data, that is, the consistency ratio should be less than the threshold value 0.1 ; if not consistent then, repeat the pair-wise comparisons. The synthesis of the priority step which needs to obtain the relative weight and global priority of alternatives by aggregating the weights over the hierarchy. After the abovementioned steps, the priority of each alternative is ranked, and a final decision can be made for selecting possibly the highest-ranked SaaS CRM solution for the company.

In addition, the team should conduct a cost-benefit analysis. The analysis aims to evaluate all feasible costs and revenues that may be acquired after the CRM solution has been configured. The outcome of the analysis will provide evidence whether the project is feasible financially, it will also reveal whether the solution 
proposed meets with the company requirement or if another project should be chased.

\subsection{Configuration Stage}

The final stage is the configuration stage. The result of stage 2 determines whether the company will go ahead with the configuration of the proposed SaaS CRM solution or not. Here, the requirement, attribute and the alternatives can be changed from stage 1 and stage 2 if any changed arises. After the final decision, the selected SaaS CRM solution will be configured with the demanded functions. Training to users is necessary and users' evaluation is expected.

\section{Case Study}

Biyemfat Enterprise is a small private real estate company based in Lagos, Nigeria and has been in the market for over 20 years. Biyemfat Enterprise is actively involved in helping their clients and individuals to develop, manage, sell, buy and lease properties. They are also involved in property and facility maintenance services which include managing shopping centres, office complexes and residential portfolios. Biyemfat Enterprises legacy systems use a spreadsheet and paper-based system for their day to day activities. This system has been in use for many years for a range of business management purposes. As the company grew, the complexity of the business processes increased, and so with the need for complex planning and information. The inefficiency and inability of the legacy system to cope with these increasing requirements resulted in the decision to implement a SaaS CRM solution.

\section{SaaS CRM Selection Method with a Case Study}

In this section, we demonstrate how to apply the proposed selection method to Biyemfat Enterprises with a view of making the right decision in their SaaS CRM solution selection process. We explain in detail how each step can be applied using the proposed selection methods in the preliminary and evaluation in Section 5.1 and Section 5.2 respectively.

\subsection{Preliminary Stage}

Since fewer people are involved in the selection process for SME, a team was formed involving the business owner (CEO), one staff person and the CRM expert with the obligation to describe the project characteristics which include the company goals, problem definition and any risk that may arise.

Secondly, the team brainstormed and identify the strategic objectives. Each similar objective was grouped together by continually asking "what do you mean by that". Asking what the identified objectives mean lead to several sub-objectives that further describe exactly what is meant by these terms. In order to ensure flexibility to any change in the business plan, several meetings were scheduled to constantly review the process. Thereafter, the following objectives were compiled:

Objective 1: To maximize customer retention

1.1. Increase customer base

1.2. Have better customer relationship

1.3. Promptly respond to customer requests

Objective 2: To revitalize company marketing strategy

2.1. Increase sales volume by $20 \%$ in 12 months

2.2. Develop and implement a promotional plan to drive increased business

Objective 3: To enhance business performance

3.1. Increase revenue by $10 \%$ annually (Marketing automation)

3.2. Reduce operating costs by $10 \%$ in 12 months

3.3. Create a flexible environment to allow the employee to work anytime, anywhere (Mobile access)

The team brainstormed on which of the criteria from literature (Table 1) is needed to achieve the stated objectives. The team evaluate each objective and criteria then concluded to have an affordable SaaS CRM solution with complete functionality, easy to use, fit with the company business strategy, have excellent system flexibility, very reliable and have a reputable vendor. Based on the team response, the selection criteria were extracted and compiled as shown in Table 2.

\begin{tabular}{|c|c|c|}
\hline Criteria & Attributes & Objectives \\
\hline Total-cost & $\begin{array}{l}\text { Affordable Subscription } \\
\text { cost }\end{array}$ & $1,2,3$ \\
\hline \multirow[t]{4}{*}{ Functionality } & Salesforce Automation & $1,2,3$ \\
\hline & Market automation & $2,2.1,3$ \\
\hline & Service automation & 1.3 \\
\hline & Analytical CRM & 1 \\
\hline \multirow[t]{2}{*}{ Usability } & Friendly GUI & $1,2,3$ \\
\hline & Mobility & 3.3 \\
\hline \multirow[t]{4}{*}{ Flexibility } & Customization & 2.2 \\
\hline & Compatibility & --- \\
\hline & Scalability & 3 \\
\hline & Ease of integration & \\
\hline \multirow[t]{2}{*}{ Reliability } & Security/ recovery & $1,2,3$ \\
\hline & Stability & \\
\hline \multirow[t]{3}{*}{ Fit with company } & Business goal & $1,2,3$ \\
\hline & Industry & \\
\hline & Capabilities & \\
\hline \multirow[t]{2}{*}{ Vendor quality } & Reputation & $1,2,3$ \\
\hline & Technical support & \\
\hline
\end{tabular}

Table 2: Mapping between objectives and different level criteria

Lastly, potential alternatives were discussed within the team to identify feasible alternatives. Several numbers of popular SaaS CRM providers that offer a wide variety of features were identified. Based on thorough examination and preliminary 
screening from different vendors via the internet, product/service release conferences, professional magazines, vendor websites, and other sources, the team was able to scrutinize two popular SaaSbased CRM solution specifically solutions that support small businesses. As a result, we were able to identify Zoho CRM and Salesforce as the two major SaaS CRM solutions in Nigeria market (Since our case study is in Nigeria). The two-software company were also reviewed based on their business workflows and special requirements. The team signs up for a demo and free trial for both Zoho CRM and Salesforce software which were used to examine the capacity of each software and its various features, without committing any valuable resources.

Thereafter, the fundamental hierarchical structure was constructed as shown in Figure 2, level 0 represents the main goal which is to select the most suitable SaaS CRM solution. Level 1 was constructed based on the criteria identified Level 1 was further broken down to determine the attribute pertinent to evaluating each SaaS CRM solution (level 2). The two alternatives (Salesforce and Zoho CRM) represent Level 3 of the fundamental hierarchy structure.

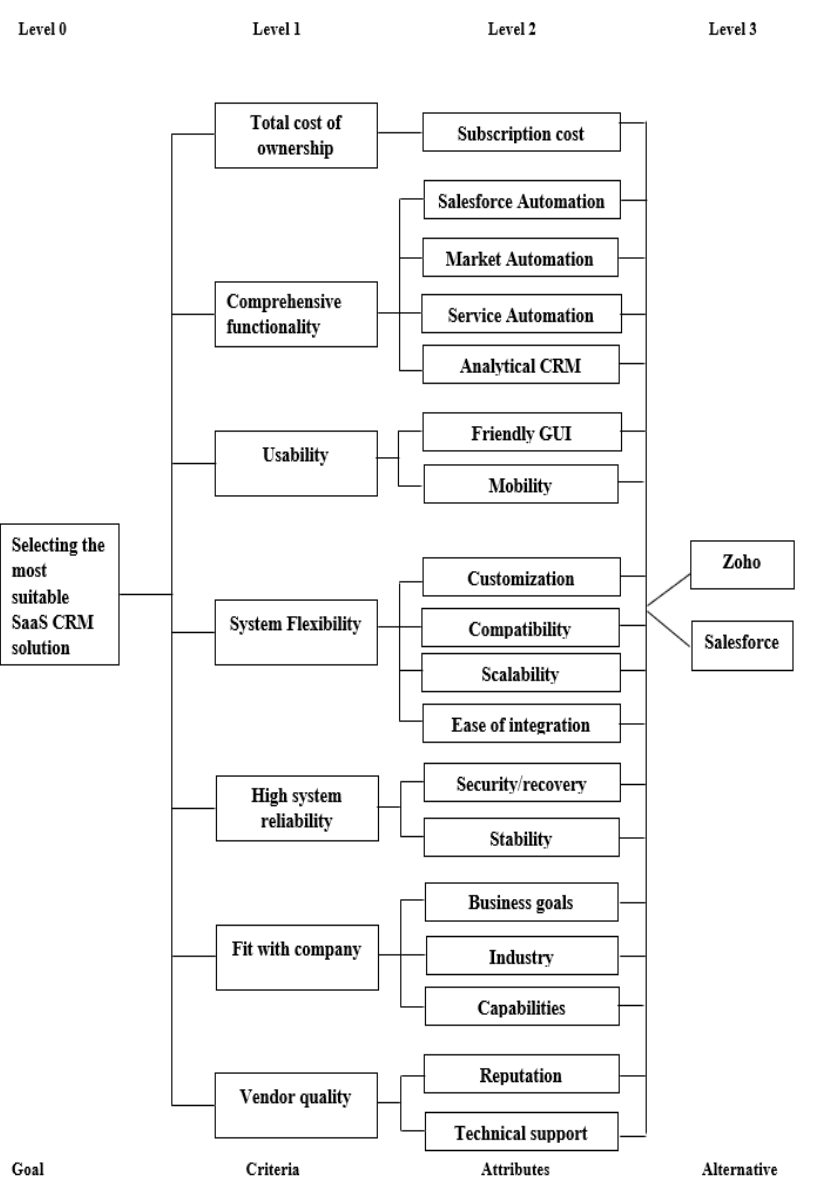

Figure 2: Fundamental Objective Hierarchy for SaaS CRM Software Selection

\subsection{Evaluation Stage}

Based on AHP methodology, the team set up an $\mathrm{n} x \mathrm{n}$ matrix or pairwise comparison matrix, where $n$ represents the number of evaluation criteria to be considered. And $C_{1}, C_{2} \ldots C_{n}$ represents a set of elements, while $\mathrm{a}_{\mathrm{ij}}$ signifies a quantified judgment on a pair of elements $\mathrm{C}_{\mathrm{i}} \mathrm{C}_{\mathrm{j}}$. The matrix $\mathrm{n} * \mathrm{n}$ is depicted below.

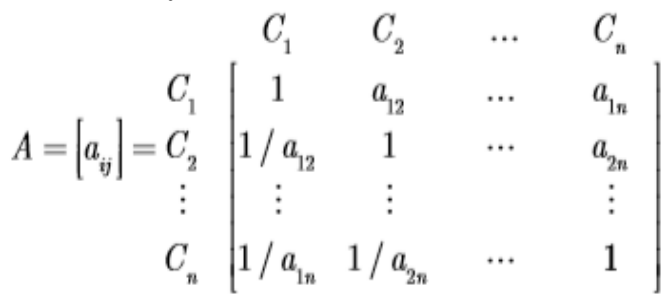

In matrix $A$, each entry $a_{i j}$ represents the importance of the ith criterion relative to the $\mathrm{jth}$ criterion. The ith criterion is preferable than the $j$ th criterion when aij $>1$. Then when $a_{i j}<1$, we say criterion less preferable than the ${ }_{\text {jth }}$ criterion. Also, if both criteria have the same importance then $\mathrm{a}_{\mathrm{ij}}=1$.

$$
a_{i j}>0, \quad a_{j i}=\frac{1}{a_{i j}}, \quad a_{i i}=1
$$

By using Saaty [34] scale ranking of $(1,3,5,7,9)$, we can determine the value of $(i, j)$ in the pairwise comparison matrix and the inverse value of the assigned number is assigned to the $(j, i)$ as display in Table 3.

\begin{tabular}{cccccccc}
\hline Criteria & $\begin{array}{c}\text { Total } \\
\text { cost }\end{array}$ & Functionality & Usability & Flexibility & Reliability & $\begin{array}{c}\text { Fit with } \\
\text { company }\end{array}$ & $\begin{array}{c}\text { Vendor } \\
\text { quality }\end{array}$ \\
\hline Total cost & 1 & $1 / 7$ & $1 / 5$ & $1 / 3$ & 2 & $1 / 3$ & 1 \\
Functionality & 7 & 1 & 3 & 4 & 9 & 3 & 6 \\
Usability & 5 & $1 / 3$ & 1 & 2 & 5 & 3 & 4 \\
Flexibility & 3 & $1 / 4$ & $1 / 2$ & 1 & 4 & 2 & 3 \\
Reliability & $1 / 2$ & $1 / 9$ & $1 / 5$ & $1 / 4$ & 1 & $1 / 4$ & $1 / 3$ \\
Fit with company & 3 & $1 / 3$ & $1 / 3$ & $1 / 2$ & 4 & 1 & 2 \\
Vendor quality & 1 & $1 / 6$ & $1 / 4$ & $1 / 3$ & 3 & $1 / 2$ & 1
\end{tabular}

Table 3: Pairwise comparison matrix

Thereafter, we calculate the relative weight and check its consistency. To calculate the relative weights, $\{w\}$, firstly, we sum up the elements in each column in Table 3. Then we normalize the matrix. This means that we divide each element in every column by the sum of its column The normalized value is displayed in Table $\mathbf{4}$ and computed using the equation below:

$$
\bar{a}_{=} \frac{a_{i j}}{\sum_{l=1}^{n} a_{l j}}
$$




\begin{tabular}{lcllllll}
\hline Criteria & Total cost & Functionality & Usability & Flexibility & Reliability & $\begin{array}{l}\text { Fit with } \\
\text { company }\end{array}$ \\
\hline Total cost & 0.049 & 0.060 & 0.036 & 0.039 & 0.071 & 0.033 & 0.058 \\
Functionality & 0.341 & 0.429 & 0.547 & 0.476 & 0.321 & 0.298 & 0.346 \\
Usability & 0.244 & 0.142 & 0.182 & 0.238 & 0.179 & 0.298 & 0.231 \\
Flexibility & 0.146 & 0.107 & 0.091 & 0.119 & 0.143 & 0.198 & 0.173 \\
Reliability & 0.024 & 0.047 & 0.036 & 0.030 & 0.036 & 0.025 & 0.019 \\
Fit with company & 0.146 & 0.142 & 0.060 & 0.059 & 0.143 & 0.099 & 0.115 \\
Vendor quality & 0.049 & 0.073 & 0.046 & 0.039 & 0.107 & 0.005 & 0.058 \\
\hline Sum of columns & 1.000 & 1.000 & 1.000 & 1.000 & 1.000 & 1.000 & 1.000 \\
\hline
\end{tabular}

Table 4: Normalized pairwise matrix

Finally, calculating the criteria weight vector $\{w\}$ is achieved by averaging the entries on each row of $\bar{a}$. This result is shown in Table 5 and computed as:

Relative weight $\{\mathbf{w}\}=\frac{\sum_{l=1}^{n} \bar{a}_{i l}}{n}$

For example

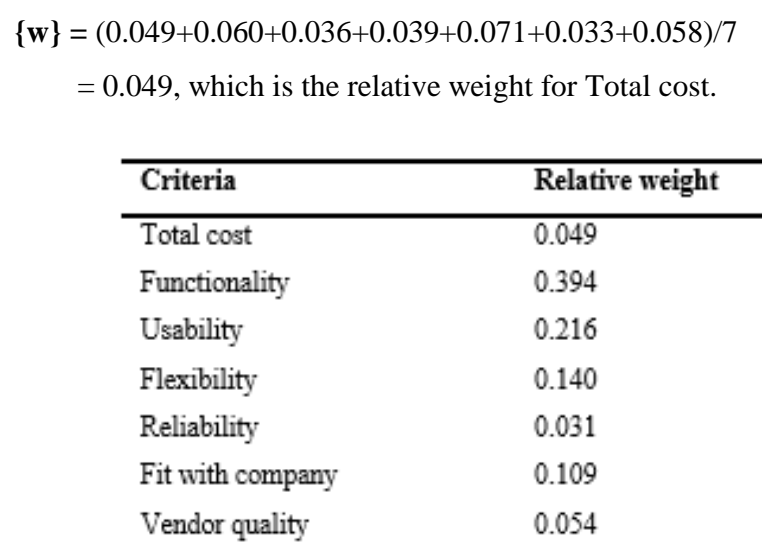

Table 5: Relative weight for matrix

The same steps were carried out for each attribute to obtain each relative weight. As displayed in Table 6, the Global weight (3) of each attribute is computed by multiplying the weight of the main criteria (1) by the corresponding attribute weighting value (2).

For example: $\quad$ Subscription cost $=0.049 * 1.000=0.049$ Salesforce automation $=0.394 * 0.530=0.209$.

With this, we were able to identify the 10 highest weighting value which is: Subscription cost, Salesforce automation, Market automation, Service automation, Friendly GUI, Mobility, Customization, Scalability, Business goal and Reputation, as shown in Table 6. These identified weighs were subsequently used to evaluate the SaaS CRM alternatives for the selection process

\begin{tabular}{|c|c|c|c|c|}
\hline Criterion & Local weight (1) & Attribute & Local weight (2) & Global weight (3) \\
\hline Total Cost & 0.049 & Subscription cost & 1.000 & 0.049 \\
\hline \multirow[t]{4}{*}{ Functionality } & 0.394 & Sales force automation & 0.530 & 0.209 \\
\hline & & Market automation & 0.230 & 0.091 \\
\hline & & Service automation & 0.170 & 0.067 \\
\hline & & Analytic CRM & 0.070 & 0.028 \\
\hline \multirow[t]{2}{*}{ Usability } & 0.216 & Friendly GUI & 0.667 & 0.144 \\
\hline & & Mobility & 0.333 & 0.071 \\
\hline \multirow[t]{4}{*}{ Flexibility } & 0.140 & Customization & 0.535 & 0.075 \\
\hline & & Compatibility & 0.053 & 0.007 \\
\hline & & Scalability & 0.268 & 0.038 \\
\hline & & Ease of integration & 0.143 & 0.020 \\
\hline \multirow[t]{2}{*}{ Reliability } & 0.031 & Security/recovery & 0.750 & 0.023 \\
\hline & & Stability & 0.250 & 0.007 \\
\hline \multirow{3}{*}{$\begin{array}{l}\text { Fit with } \\
\text { company }\end{array}$} & 0.109 & Business goal & 0.727 & 0.079 \\
\hline & & Industry & 0.080 & 0.009 \\
\hline & & Capabilities & 0.193 & 0.021 \\
\hline \multirow{2}{*}{$\begin{array}{l}\text { Vendor } \\
\text { quality }\end{array}$} & 0.054 & Reputation & 0.750 & 0.041 \\
\hline & & Technical support & 0.250 & 0.014 \\
\hline
\end{tabular}

Table 6: Final Weights of Each Criterion

Table 7 shows how the two alternatives were ranked based on their overall priorities. The result reveals that Zoho CRM appears to be the most suitable SaaS CRM for Biyemfat Enterprise, with an overall priority of $\mathbf{0 . 5 6 0}$.

The criteria weighing was carried out by the CRM expert as they have adequate knowledge of what the company wants to achieve. Hence, the number of responses is limited. The analyses consist of three phases. The first phase covers the prioritization of criteria's and attributes, while the second phase is about an alternate comparison. The third part combines the results obtained from the first two parts to rank the best SaaS CRM solution. The consistency index of each paired comparison matrix was checked throughout the evaluation process. According to Saaty [34] data remains valid or acceptable if the consistency ratio is $<0.1$. Since the consistency ratio is $0.03<0.1$. then the pairwise comparison is consistent and accepted. 


\begin{tabular}{|c|c|c|c|c|c|c|c|}
\hline \multirow[t]{2}{*}{ Criterion } & \multirow[t]{2}{*}{ Local weight (1) } & \multirow[t]{2}{*}{ Attribute } & \multirow[t]{2}{*}{ Local weight (2) } & \multicolumn{2}{|c|}{ Local weight (3) } & \multicolumn{2}{|c|}{ Global weight (4) } \\
\hline & & & & salesforce & Zoho & Salesforce & Zoho \\
\hline Total Cost & 0.049 & Subscription cost & 1.000 & 0.167 & 0.833 & 0.008 & 0.041 \\
\hline \multirow[t]{3}{*}{ Functionality } & 0.394 & Sales force automation & 0.530 & 0.249 & 0.751 & 0.052 & 0.157 \\
\hline & & Market automation & 0.230 & 0.249 & 0.751 & 0.045 & 0.045 \\
\hline & & Service automation & 0.170 & 0.333 & 0.667 & 0.022 & 0.045 \\
\hline \multirow[t]{2}{*}{ Usability } & 0.216 & Friendly GUI & 0.667 & 0.249 & 0.751 & 0.036 & 0.108 \\
\hline & & Mobility & 0.333 & 0.500 & 0.500 & 0.036 & 0.036 \\
\hline \multirow[t]{2}{*}{ Flexibility } & 0.140 & Customization & 0.535 & 0.249 & 0.751 & 0.019 & 0.056 \\
\hline & & Scalability & 0.268 & 0.667 & 0.333 & 0.025 & 0.012 \\
\hline $\begin{array}{l}\text { Fit with } \\
\text { company }\end{array}$ & 0.109 & Business goal & 0.727 & 0.333 & 0.667 & 0.026 & 0.053 \\
\hline \multirow[t]{3}{*}{ Vendor quality } & 0.054 & Reputation & 0.750 & 0.833 & 0.167 & 0.034 & 0.007 \\
\hline & & & & \multirow{2}{*}{\multicolumn{2}{|c|}{$\begin{array}{l}\text { Overall priority } \\
\text { Rank }\end{array}$}} & 0.303 & 0.560 \\
\hline & & & & & & 2 & 1 \\
\hline
\end{tabular}

Table 7: Overall rating for alternatives using AHP

\subsubsection{Cost-Benefit Analysis}

The cost-benefit analysis is a systematic process for businesses to support the decision-making process by guiding the manager to make appropriate and profitable decisions about Information system investment [35]. In this study, the cost-benefit analysis process initially involves compiling a comprehensive list detailing all costs and benefits related to the SaaS CRM configuration. Afterwards, a common unit of monetary measurement was applied to all the identified items on the list. To avoid any biased tendencies such as underestimating cost or overestimating benefit, the team took a careful approach with a conscious effort when assigning value to both costs and benefits.

Presently, the cost of personnel, stationaries, transportation and communication cost will be 3,150,000NGN, 785,200NGN, 591,120 NGN and 60,000NGN respectively without a SaaS CRM solution for 5years. Based on the discussion with the company $\mathrm{CEO}$, Biyemfat uses a paper-based system for their day to day activities which lead to high paper waste and as a result costs both time and money. Spending both time and money on administrating paper will deprive customers of having exceptional customer service. For instance, if Biyemfat Enterprise employee spends a minimum of 2 hours per day to access, retrieve and share information, work on paper documentation, putting together latest sales figure on excel sheet and or making individual templates for sales offers or advertising. That means the company loses the totals of 10hours per day from the 5 employees which are equivalent to $3020 \mathrm{NGN}$ per day and $785,200 \mathrm{NGN}$ for a whole year. This wasted cost can be invested in another project that can bring more profit to the company. With Zoho CRM, the company will improve its business processes by having quick access to the right information and make a better and informed decision. Staff will also spend less time looking for information, give better service delivery for customers and cost savings from less creation, retrieval, storing and handling of paper records. Our result reveals that Biyemfat

Enterprise can save 17,042,831.38NGN in 5 years. In addition, with effective SaaS CRM solution in place, Biyemfat will be able to save cost, automate business processes, revitalize customer strategy and improve the quality of the customer service. For instance, when a customer gets a better service and as a result, satisfaction peaks then company can easily get leads through referrals from their customers which is all free. In addition, every employee can easily access customer information as they are all stores in a single database. Employees do not have to waste time to run around to source for a single piece of information. However, it should be noted that the cost-benefit analysis has several areas for further investigation. In the analysis conducted, there are certain internal costs that can come up later in the future which may be required to have a successful transition to a new technology such as upgrade cost, training cost and vendor support.

\section{Conclusion}

This study presents a comprehensive decision-making framework for SaaS CRM evaluation and selection approach for SME using the Analytical Hierarchical Process. The SaaS CRM selection framework was developed with careful considerations ensuring the identified objective is in accordance with company business strategies. Though the exactness with which the team could provide a paired comparison was limited by their experience, 
knowledge and the complexity of CRM selection problem. As a result of this, the team was trained clearly understand the details, strength and limitation of the AHP method. It is worth noting that every organisation's business processes vary, as a result, our study is limited to a specific company requirement and the criteria identified cannot be generalized. Consistency checks were also conducted during the evaluation process to ensure the team was not biased during the assessment. The proposed method was applied to one organisation. It would be useful to apply the method into different organisations to improve the method

The result of this study has a great impact on academic and industry in terms of SaaS CRM selection. The proposed framework can be easily adopted by managers when they encounter problems in selecting a suitable SaaS CRM solution that can fit into their business strategy. The hierarchy structure developed could serve as a benchmark for the first step of establishing what criteria to be identified for SaaS CRM selection process. It can also be used by practitioners to assess their SaaS CRM software portfolio. Each of the alternatives can be substituted with another alternative depending on their needs. Based on its flexibility, the developed framework can also be customized to develop a new framework by adding new criteria that are specific to their business requirement. And lastly, the study can also be used as a reference point for the academic community during the system selection process. With this study, the researcher hopes to lessen readers' time and effort in examining and selecting appropriate SaaS CRM solution for their organization.

Future research will continue to follow up the configuration stage of the selection process for Biyemfat Enterprise for the coming 5 years. The possible changes in selection criteria will be considered and adopted according to the changes.

\section{Acknowledgements}

This research is partially funded by the State Key Research and Development Program of China (2017YFE0118700) and it is part of the FIRST project which has received funding from the European Union's Horizon 2020 research and innovation programme under the Marie Skłodowska-Curie grant agreement No. 734599. The authors will like to appreciate Biyemfat Enterprise for their support throughout the completion of this paper. We would also like to thank anonymous reviewers for their insightful comments and suggestions.

\section{REFERENCES}

[1] M. Bonacchi and P. Perego, "Improving Profitability with Customer-Centric Strategies: The Case of a Mobile Content Provider," Strategic Change, vol. 20, pp. 253-267, 2011.

[2] A. Fahed and A. Maged, "The Effect of Organizaional Culture on CRM Success," in European, Mediterranean \& Middle Eastern Conference on Information Systems , Windsor, United Kingdom, 2013.

[3] R. Rahimi and M. Kozak, "Impact of Customer Relationship Management on Customer Satisfaction: The Case of a
Budget Hotel Chain," Journal of Travel \& Tourism Marketing, vol. 34, pp. 40-51, 2017.

[4] A. Benlian and T. Hess, "Opportunities and risks of software-as-a-service: findings from a survey of IT executives," Decis Support Syst, vol. 52, pp. 232-246, 2011.

[5] Y. Peng, G. Kou, G. Wang and Y. Shi, "AMCDM: a fusion approach of MCDM methods to rank multiclass classification algorithms," Omega, vol. 39, no. 6, pp. 677689, 2011.

[6] V. M. Araujo, J. A. Vazquez and C. M. Perez, "A Framework for the Evaluation of SaaS Impact," International Journal in Foundations of Computer Science \& Technology (IJFCST), vol. 4, no. 3, 2014.

[7] S. Xiao and G. Cheng, "Application research of CRM based on SaaS," in International Conference on E-Business and EGovernment, USA, CHINA, 2010.

[8] S. H. Mohamad, N. A. Othman, J. Jabar and I. Abdul Majid, "Customer Relationship Management Practices: The Impact of Organizational Performance in SMEs of Food Manufacturing Industry," European Journal of Business and Management, vol. 6, no. 13, pp. 31-43, 2014.

[9] A. Souri, P. Asghari and R. Rezaei, "Software as a Service based CRM Providers in the Cloud Computing: Challenges and Technical Issues," Journal of Service Science Research, vol. 9, pp. 219-237, 2017.

[10] A. S. Jadhav and R. M. Sonar, "Evaluating and Selecting Software Package: A Review," Information and Software Technology, vol. 51, pp. 555-563, 2009.

[11] H. Kyung-Kwon and K. Young-Gul, Information and Management, vol. 40, pp. 25-40, 2002.

[12] A. Teltumbde, "A framework for evaluating ERP projects," International Journal of Production Research, vol. 38, no. 17, pp. 4507-4520, 2000.

[13] M. M. Miruna, M. Constanţa and N.-A. Gabrieoa, "Why should SME adopt IT enabled CRM strategy?," Academy of Economic Studies, vol. 1, no. 41, pp. 109-112, 2007.

[14] L. Cricelli, M. F. Federico, G. Marco and G. Michele, "Searching for the one: Customer relationship management software selection," J Multi-Crit Decis Anal., pp. 1-6, 2019.

[15] E. Colombo and C. Francalanci, "Selecting CRM packages based on architectural, functional, and cost requirements: empirical validation of a hierarchical ranking model.," Requirement Engineering, vol. 9, pp. 186-203, 2004.

[16] B. Öztayşi, T. Kaya and C. Kahraman, "Performance comparison based on customer relationship management using analytic network process," Expert Systems with Applications: An International Journal, vol. 38, no. 8, pp. 9788-9798, 2011.

[17] A. Keramati and M. S. Sangari, "A Success Framework to Investigate Critical Factors Associated with Implementation of Customer Relationship Management: A Fuzzy ANP Approach," International Journal of Customer Relationship Marketing and Management, vol. 2, no. 2, 2011.

[18] Y. C. Lee, N. H. Tang and V. Sugumaran, "pen source CRM software selection using the analytic hierarchy process.," Information System Management, vol. 31, no. 1, pp. 2-20, 
2014.

[19] R. E. M, Diffusion of Innovations,, 5th Edition ed., Free Press, 2003.

[20] C.-J. Guo, W. Sun, Z.-B. Jiang, Y. Huang, B. Gao and Z.-H. Wang, "Study of Software as a Service Support Platform for Small and Medium Businesses," New Frontiers in Information and Software as Services, vol. 74, pp. 1-30, 2011.

[21] G. Manish and M. Shrikant, "An Approach for Selecting Software-as-a-Service (SaaS) Product," in 2009 IEEE International Conference on Cloud Computing, Bangalore, India, 2009.

[22] J. Repschlaeger, W. Stefan, Z. Ruediger and T. Klaus, "Selection Criteria for Software as a Service: An Explorative Analysis of Provider Requirements," in Proceedings of the Eighteenth Americas Conference on Information System, Seattle, Washington, 2012.

[23] Y. C. Lee, N. H. Tang and V. Sugumaran, "pen source CRM software selection using the analytic hierarchy process.," Information System Management, vol. 31, no. 1, pp. 2-20, 2014.

[24] M. Keil and A. Tiwana, ". Relative importance of evaluation criteria for enterprise systems: A conjoint study.," Information Systems Journal, vol. 16, pp. 237-262, 2006.

[25] L. Cricelli, M. F. Federico, G. Marco and G. Michele, "Searching for the one: Customer relationship management software selection," J Multi-Crit Decis Anal., pp. 1-6, 2019.

[26] F. D. F. Nemésio, I. P. S. P. Clarindo, L. Z. André and H. S. B. Paulo, "Quality attributes for SaaS's products," in 5th Iberian Conference on Information Systems and Technologies, Santiago de Compostela, Spain, 2010.

[27] M. Lin, "A study of mainstream features of CRM system and evaluation criteria," in Proceedings of the 2003 American Society for Engineering Education Annual Conference \& Exposition. American Society for Engineering Education., 2003.

[28] C. G. Sen and H. Baraçl1, "Fuzzy quality function deployment based methodology for acquiring enterprise software selection requirements," Expert Systems with Applications, vol. 37, pp. 3415-3426, 2010.

[29] I. Friedrich and M. H. Breitner, "Towards a process model for efficient customer relationship management system selection," in Digitale Bibliothek, Braunschweig, 2012.

[30] L. Jae Yoo, W. L. Jung, C. Du Wan and D. K. Soo, "A Quality Model for Evaluating Software-as-a-Service in Cloud Computing," in 2009 Seventh ACIS International Conference on Software Engineering Research, Management and Applications, Haikou, China, 2009.

[31] G. Manish and M. Shrikant, "An Approach for Selecting Software-as-a-Service (SaaS) Product," in 2009 IEEE International Conference on Cloud Computing, Bangalore, India, 2009.

[32] E. W. Bernroider and S. Koch, "Differences in Characteristics of the ERP System Selection Process between Small or Medium and Large Organizations," in Sixth Americas Conference on Information Systems (AMCIS), 2000.
[33] C.-C. Wei, C.-F. ,. Chien and M.-J. J. Wang, "selection, An AHP-based approach to ERP system," International journal of Economies, vol. 96, pp. 47-62, 2005.

[34] T. L. Saaty, Decision making for leaders:The analytic hierarchy process for decisions in a complex world., Pittsburgh, PA: RWS Publications, 2001.

[35] J. King and E. Schrems, "Cost-Benefit Analysis in Information Systems Development and Operation," ACM Computing Surveys, vol. 10, no. 1, pp. 19-34, 1978.

[36] C.-J. Guo, W. Sun, Z.-B. Jiang, Y. Huang, B. Gao and Z.-H. Wang, "Study of Software as a Service Support Platform for Small and Medium Businesses," New Frontiers in Information and Software as Services, vol. 74, pp. 1-30, 2011.

[37] M. Lin, "A study of mainstream features of CRM system and evaluation criteria," in Proceedings of the 2003 American Society for Engineering Education Annual Conference \& Exposition. American Society for Engineering Education., 2003.

[38] L. Jae Yoo, W. L. Jung, C. Du Wan and D. K. Soo, "A Quality Model for Evaluating Software-as-a-Service in Cloud Computing," in 2009 Seventh ACIS International Conference on Software Engineering Research, Management and Applications, Haikou, China, 2009.

[39] R. E. M, Diffusion of Innovations,, 5th Edition ed., Free Press, 2003.

[40] M. Keil and A. Tiwana, ". Relative importance of evaluation criteria for enterprise systems: A conjoint study.," Information Systems Journal, vol. 16, pp. 237-262, 2006.

[41] C. G. Sen and H. Baraçl1, "Fuzzy quality function deployment based methodology for acquiring enterprise software selection requirements," Expert Systems with Applications, vol. 37, pp. 3415-3426, 2010.

[42] I. Friedrich and M. H. Breitner, "Towards a process model for efficient customer relationship management system selection," in Digitale Bibliothek, Braunschweig, 2012.

[43] J. Repschlaeger, W. Stefan, Z. Ruediger and T. Klaus, "Selection Criteria for Software as a Service: An Explorative Analysis of Provider Requirements," in Proceedings of the Eighteenth Americas Conference on Information System, Seattle, Washington, 2012.

[44] F. D. F. Nemésio, I. P. S. P. Clarindo, L. Z. André and H. S. B. Paulo, "Quality attributes for SaaS's products," in 5th Iberian Conference on Information Systems and Technologies, Santiago de Compostela, Spain, 2010. 